INPLASY

PROTOCOL

To cite: Wang et al.

Comparison of effectiveness on interventional therapies for unresectable hepatic cancer- a network meta-analysis. Inplasy protocol 202090030. doi:

10.37766/inplasy2020.9.0030

Received: 07 September 2020

Published: 07 September 2020

Corresponding author:

Jiazheng Liu

59904698@qq.com

Author Affiliation:

The Fourth Affiliated Hospital of China Medical University

Support: None.

Review Stage at time of this submission: Data analysis.

Conflicts of interest:

None.

\section{Comparison of effectiveness on interventional therapies for unresectable hepatic cancer - a network meta-analysis}

\author{
Wang, S1; Liu, D²; Wei, H³; Shi, G4; Liu, J5.
}

Review question / Objective: For individuals diagnosed with unresectable hepatic cancer, does interventional treatment including TACE, Hepatic artery infusion chemotherapy, chemoembolization, radio thermal therapy in combination with small molecular drugs such as sorafenib or other possible drugs improve the overall prognosis of interventional group in comparison with placebo or other therapy, which one is the best therapy?

Condition being studied: This meta-analysis will look at intervention measures on unresectable hepatic cancer.

INPLASY registration number: This protocol was registered with the International Platform of Registered Systematic Review and Meta-Analysis Protocols (INPLASY) on 07 September 2020 and was last updated on 07 September 2020 (registration number INPLASY202090030).

\section{INTRODUCTION}

Review question / Objective: For individuals diagnosed with unresectable hepatic cancer, does interventional treatment including TACE, Hepatic artery infusion chemotherapy, chemoembolization, radio thermal therapy in combination with small molecular drugs such as sorafenib or other possible drugs improve the overall prognosis of interventional group in comparison with placebo or other therapy, which one is the best therapy?

Condition being studied: This metaanalysis will look at intervention measures on unresectable hepatic cancer. 


\section{METHODS}

Participant or population: The study population will be limited to individuals with confirmed diagnosis of unresectable hepatic cancer, patient aged between 18-70 years.

Intervention: The interventions of interest are hepatic artery infusion chemotherapy, transcatheter artery chemoembolization (TACE), hepatic artery infusion chemotherapy in combination with sorafenib, transcatheter artery chemoembolization (TACE) in combination with sorafenib, and any other interventional therapies on the treatment of advanced or unresectable liver cancer.

Comparator: Inclusion criteria and study selection To be included in this metaanalysis, studies must meet the following inclusion criteria: (1) study design: randomized controlled trials (RCTs) and quasi-RCTs (2) population: adult patients who were histologically and/or clinically diagnosed HCC; (3) intervention: neoadjuvant HAIC; , transcatheter artery chemoembolization (TACE), hepatic artery infusion chemotherapy in combination with sorafenib, transcatheter artery chemoembolization (TACE) in combination with sorafenib, and any other interventional therapies on the treatment of advanced or unresectable liver cancer comparison: other therapy methods; (4) outcomes: provided 1 of the following outcomes of interest: OS, DFS, ORR, DCR, or complications. When several publications from the same trial were presented, we only included the one with the most complete data, or both if they had different outcome measures. Studies were excluded if they were non-comparative studies, or case report, case series, or did not apply HAIC for HCC patients, or did not report the data of our interest.

Study designs to be included: Randomized controlled trials (RCTs) and quasi-RCTs.

Eligibility criteria: Inclusion criteria (1) study design: randomized controlled trials (RCTs) and quasi-RCTs (2) population: adult patients who were histologically and/or clinically diagnosed HCC; (3) intervention: neoadjuvant HAIC; , transcatheter artery chemoembolization (TACE), hepatic artery infusion chemotherapy in combination with sorafenib, transcatheter artery chemoembolization (TACE) in combination with sorafenib, and any other interventional therapies on the treatment of advanced or unresectable liver cancer comparison: other therapy methods; (4) outcomes: provided 1 of the following outcomes of interest: OS, DFS, ORR, DCR, or complications. Exclusion criteria 1. noncomparative studies, or case report, case series, 2. did not apply HAIC for HCC patients, 3. did not report the data of our interest.

Information sources: Pubmed, EMBASE, CINHAL, CENTRAL, CNKI, google scholar, if full text could not be available online, contact with authors, trial registers including central, clinical trials will also be searched for design information.

Main outcome(s): The main outcome of included studies was overall survival and Disease-free survival. Measurements of effects, they were measured by hazard ratio.

Data management: The Cochrane Risk of Bias Tool will be used to evaluate study quality. This tool assessed studies on seven criteria. These include random sequence generation, allocation concealment, blinding of participants and personnel. blinding of outcome assessment, incomplete outcome data, selective reporting, and other bias.

Strategy of data synthesis: A random effects meta-analysis will be performed in to determine the pooled effect in terms of weighted mean difference (WMDs) and their $95 \%$ confidence intervals (95\% Cls) between the different study groups. We tested for heterogeneity with the Cochran $Q$-test and $I^{2}$ statistic to measure inconsistency of treatment effects across studies. Publication bias will be assessed 
using a funnel plot and Egger's test. Heterogeneity and subgroup analysis will be done, to help with confounders where possible. We will use trial sequential analysis methodology to avoid inflation of type I error due to multiplicity of analysis. We will adapt this methodology to the special characteristics of the review question but maintaining the same aim of efficiently controlling type I error inflation.

Subgroup analysis: No planned analysis of subgroups, we will develop subgroup analysis according to the planned metaregression.

Sensibility analysis: We tested for heterogeneity with the Cochran Q-test and $I^{2}$ statistic to measure inconsistency of treatment effects across studies. Publication bias will be assessed using a funnel plot and Egger's test. Heterogeneity and subgroup analysis will be done, to help with confounders where possible. We will use trial sequential analysis methodology to avoid inflation of type I error due to multiplicity of analysis. We will adapt this methodology to the special characteristics of the review question but maintaining the same aim of efficiently controlling type I error inflation.

Language: English.

Country(ies) involved: China.

Keywords: unresectable hepatic cancer, HAIC, TACE, small molecular drugs, Overall survival, PFS, network meta.

Contributions of each author:

Author 1 - Shaolei Wang - Author 1 drafted the manuscript.

Author 2 - Dehua Liu - The author provided statistical expertise.

Author 3 - Hong Wei - The author contributed to the development of the selection criteria, and the risk of bias assessment strategy.

Author 4 - Guodong Shi - The author read, provided feedback and approved the final manuscript.
Author 5 - Jiazheng Liu - The author read, provided feedback and approved the final manuscript. 